\title{
Movable dynamic grating-based optical delay line in polarization maintaining fibers
}

\author{
Sanghoon Chin, Nikolay Primerov and Luc Thévenaz \\ Ecole Polytechnique Fédérale de Lausanne, STI-GR-SCI-LT, Station 11, CH-1015 Lausanne, Switzerland \\ sanghoon.chin@epfl.ch
}

\begin{abstract}
A new type of all optical delay line is realized in fibers. A local dynamic grating reflector can be generated everywhere in the fiber, demonstrating $>1 \mu$ s delay for 650 ps pulses. OCIS codes: (060.4370) Nonlinear optics, fibers; (290.5900) Scattering, stimulated Brillouin; (060.3735) Fiber Bragg gratings
\end{abstract}

The development of all-optical delay lines using various approaches has been considered as a priority by the photonics community. Optical systems providing continuously tunable signal delaying turn out to be crucial functions in optical communication systems and phased array antennas. Various schemes to produce a high capacity optical buffering have been experimentally demonstrated, using diverse physical phenomena in optical fibers such as slow and fast light, dispersive delay line and fiber Bragg grating [1]. However, it is interesting to mention that all these techniques share one common principle to generate time delays, namely the dynamic control of the group velocity of a light signal propagating through a dispersive optical medium. This approach causes inevitable signal distortion accompanying the signal delaying. Actually, signal pulses suffer from the effect of intrinsic or induced chromatic dispersion present in the delaying device, resulting in a substantial delay-dependent pulse broadening.

In this paper, we propose a novel architecture to provide continuously tunable optical signal delaying with an ideally fast reconfigurable time, equal to the preset optical delay that constitutes the theoretical limit fixed by causality. The signal delaying mechanism in this technique relies on the fact that dynamic acousto-optic gratings produced by the stimulated Brillouin process (SBS) can be created and localized at any desired position along a highly birefringent fiber. Fig. 1 depicts the schematic diagram of this new design for an optical delay line, realized in polarization maintaining fibers (PMF). Our delaying system is actually based on two distinct physical processes. First, a localized acoustic wave is generated in the PMF, sustained by the SBS interaction between two pump pulses propagating in the fiber along one principal polarization axis [2]. This acoustic wave then simply plays the role of a grating reflector. Second, the incident signal, which is at an optical frequency well distinct to the two pump pulses, propagates along the orthogonal polarization axis and experiences a partial reflection by the generated grating [3]. Since the acoustic grating can only be built up where the SBS interaction between the two pump pulses takes place, it can be flexibly created at any preset position over the entire length of the PMF, simply by retiming Pump 1 pulse. Using this principle an isolated signal pulses with FWHM duration of 650 ps was continuously delayed up to 1.15 $\mu$ s through a $120 \mathrm{~m}$ long PMF, equivalent to a fractional delay of 1769 . This system is also suitable for a real data bit sequence, but with a maximum delay limited by the temporal decay of the acoustic wave. Fig. 2 shows the time waveforms of a reflected pulse train while the grating was shifted along the fiber by $100 \mathrm{ps}$ steps, resulting in continuous time delays up to 6 ns for 280 ps pulses (fractional delay of 47) with no significant distortion.

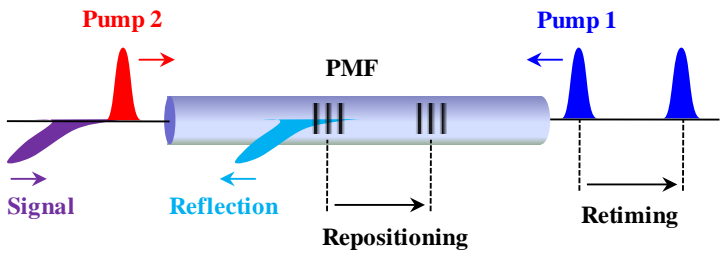

Fig. 1. Principle to generate tunable time delays in a PMF, by simply displacing the grating position, so varying the round-trip time through the fiber that the signal pulse experiences.

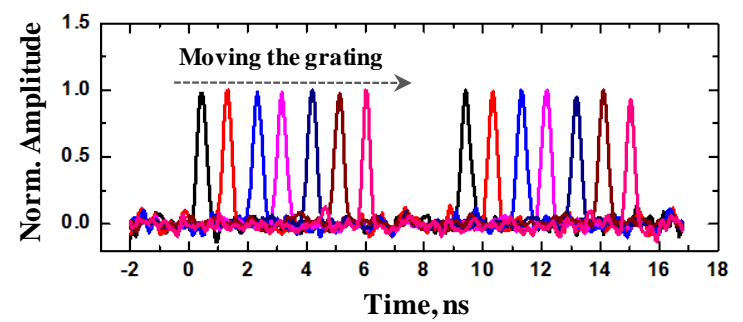

Fig. 2. Selected time traces of delayed signal pulse trains while shifting the position of dynamic grating by 100 ps steps.

\section{References}

[1] J. B. Khurgin and R. S. Tucker, Slow Light: Science and Applications, (Boca Raton, CRS Press 2009).

[2] Z. Zhu, D. J. Gauthier, and R.W. Boyd, "Stored light in an optical fiber via stimulated Brillouin scattering," Science 318, 1748-1750 (2007).

[3] K. Y. Song, W. Zou, Z. He, and K. Hotate, "All-optical dynamic grating generation based on Brillouin scattering in polarization maintaining fiber," Opt. Lett. 33, 926-928 (2008). 